FUNDAMENTALS IN CHEMICAL PHYSICS 


\section{Fundamentals in Chemical Physics}

by

Franco Battaglia

Department of Physics 'Edouardo Amaldi', Università degli Studi 'Roma Tre',

Rome, Italy

and

Thomas F. George

Department of Chemistry,

University of Wisconsin at Stevens Point,

Stevens Point, Wisconsin, U.S.A. 
A C.I.P. Catalogue record for this book is available from the Library of Congress.

ISBN 978-90-481-5082-3ＩSBN 978-94-017-1636-9 (eBook)

DOI $10.1007 / 978-94-017-1636-9$

Printed on acid-free paper

This is a completely revised and updated translation of the original Italian work Lezoine de Chimica Fisica by

F. Battaglia, CEDAM, Padova, 1997.

Translated by the authors.

\section{All Rights Reserved}

(C) 1998Springer Science+Business Media Dordrecht Originally published by Kluwer Academic Publishers in 1998

No part of the material protected by this copyright notice may be reproduced or utilized in any form or by any means, electronic or mechanical, including photocopying, recording or by any information storage and retrieval system, without written permission from the copyright owner. 


\section{Table of Contents}

Introduction $\quad$ ix

1 Classical Physics 1

1.1 Newtonian mechanics ................ . . . . 1

1.2 Lagrangian mechanics ................ 2

1.3 Hamiltonian mechanics . . . . . . . . . . . . . . 4

1.4 Constants of the motion ............. 5

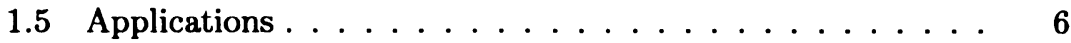

1.5.1 Harmonic oscillator . . . . . . . . . . . . 6

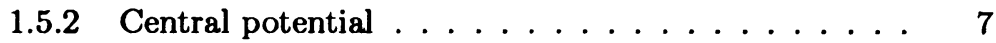

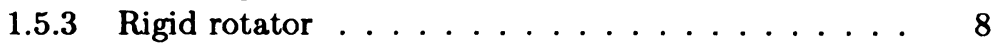

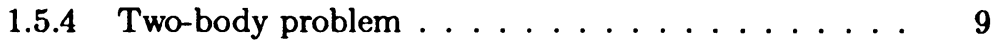

1.6 Electrodynamics . . . . . . . . . . . . . 10

2 Quantum Physics $\quad 17$

2.1 Mathematical formalism . . . . . . . . . . . . 18

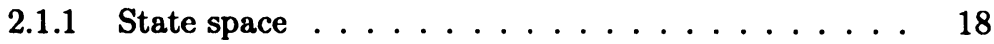

2.1.2 Linear operators .............. 21

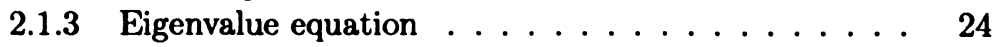

2.1.4 Observables ................ 26

2.2 Physical interpretation . . . . . . . . . . . 27

2.2.1 Probabilistic interpretation .......... 28

2.2.2 Coordinate representation ........... 31

2.2.3 Schrödinger equations . . . . . . . . . . . 33

2.2.4 Angular momentum ............. 35

2.2 .5 Many-body systems ............. 41

2.3 Applications ................... 43

2.3.1 One-dimensional system . . . . . . . . . . 43

2.3.2 Free particle ................ 44

2.3.3 Particle in a box ............. 45 
2.3.4 Harmonic oscillator ............... 47

2.3.5 Rigid rotator ............... 49

2.3.6 Central potential ............. 49

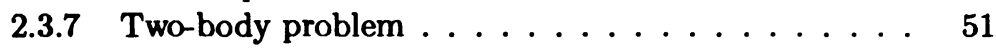

2.4 Approximation methods ............... 51

2.4.1 Variational method .............. 51

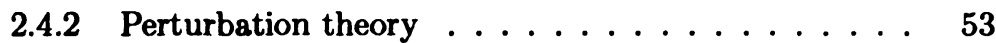

2.5 Electrodynamics ................ 57

3 Thermodynamics 63

3.1 Internal energy and entropy .............. 63

3.2 Intensive variables . . . . . . . . . . . . 67

3.3 Partition functions .................. 71

3.3.1 Canonical ensemble ............. 71

3.3.2 Isothermal-isobaric ensemble .......... 73

3.3.3 Grand canonical ensemble . . . . . . . . . . . . 74

3.4 Thermodynamic potentials ............ 75

3.4.1 Helmholtz free energy ............ 76

3.4.2 Gibbs free energy .............. 77

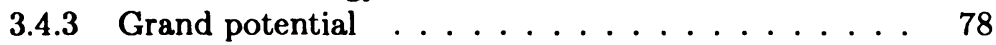

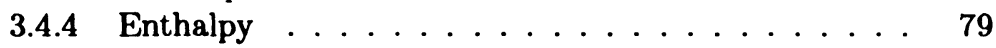

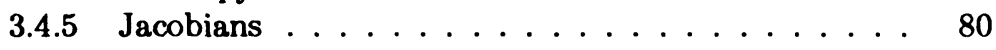

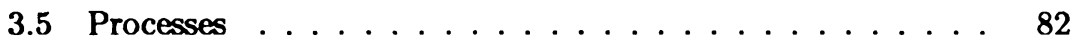

3.5.1 Equilibrium ................. 82

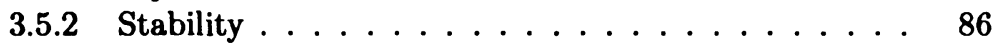

3.5.3 Work and heat ..................... 89

3.5.4 Principles of thermodynamics .......... 93

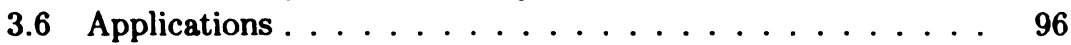

3.6.1 Equipartition theorem ............ 96

3.6.2 Ideal crystal . . . . . . . . . . . . . . . . . 98

3.6 .3 Ideal quantum gases . . . . . . . . . . . . . 103

3.6.4 Ideal classical gas . . . . . . . . . . . . . 118

3.6.5 Examples of processes ............. 125

3.6.6 Mixture of ideal gases .............. 133

4 Quantum Chemistry 141

4.1 Hydrogen-like atom . . . . . . . . . . . . . . . . . . 142

4.2 Many-electron atoms . . . . . . . . . . . . . . . 148

4.3 Electronic structure of molecules . . . . . . . . . . 152

4.3.1 Diatomic molecules .............. 163

4.3.2 Polyatomic molecules . . . . . . . . . . 166

4.4 Nuclear motion in molecules . . . . . . . . . . . . . 169 
TABLE OF CONTENTS vii

4.4.1 Diatomic molecules . . . . . . . . . . . . . . 169

4.4.2 Polyatomic molecules . . . . . . . . . . . 174

5 Molecular Spectroscopy 183

5.1 Microwave spectroscopy . . . . . . . . . . . . . 189

5.2 IR spectroscopy . . . . . . . . . . . . . . . . 192

5.3 UV spectroscopy ................... 195

5.4 Raman spectroscopy ................ 200

5.5 NMR spectroscopy ................. 203

5.5.1 Chemical shift .............. 206

5.5 .2 Spin-spin coupling .............. 208

5.5.3 First-order spectra . . . . . . . . . . . 216

5.5 .4 Conclusions ................ 218

6 States of Aggregation $\quad 221$

6.1 Real gases . . . . . . . . . . . . . . . . . 221

6.1.1 Intramolecular structure . . . . . . . . . . 221

6.1.2 Intermolecular interactions . . . . . . . . 232

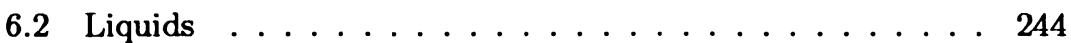

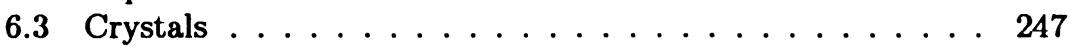

6.3.1 Lattice structure . . . . . . . . . . . . 247

6.3.2 Electronic structure ............. 252

6.4 Phase equilibria . . . . . . . . . . . . . . 257

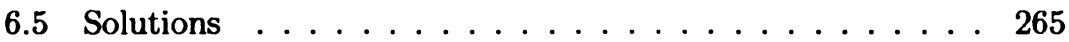

7 Chemical Reactions $\quad 275$

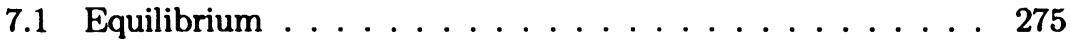

7.2 Kinetics ..................... 283

7.2.1 Descriptive kinetics . . . . . . . . . . . 283

7.2.2 Reaction mechanisms ............ 290

7.2 .3 Conclusions ................. 302

$\begin{array}{lr}\text { Index } & \mathbf{3 0 7}\end{array}$ 


\section{Introduction}

Each science has its own fundamental program to pursue, although the fields pertaining to a given science overlap those in another in a wide variety of interdisciplinary ways. In this regard, the fundamental program of chemical physics consists in understanding chemical phenomena in terms of the most fundamental laws of physics. The purpose just stated can be pursued by adopting two major methodologies - an experimental and a theoretical one. The place of action for experimentalists is a laboratory, where they try to keep under controlled and repeatable conditions the chemical phenomenon to be studied. The place of action for theoreticians is a desk (possibly equipped with computing facilities), where they try either to fit a chemical phenomenon within the known physical theories, or to tailor a specific theory for it from which emerges the essential features that make the phenomenon intelligible.

Besides their own technical skills, both experimentalists and theoreticians need a clear understanding, at a quantitative level, of the fundamental ideas of the subject. Chemical physics is a science on its own. Its main concern is chemistry, whose phenomena it wishes to describe using the language of physics and mathematics. In this book we shall not consider any of the tools of a chemical physicist, i.e., laboratory apparata or theoretical techniques. Rather, we shall focus on concepts, which are presented at the quantitative level, i.e., carefully discussing the equations (their origin and strength of predictability) governing the phenomena of chemical-physics interest.

We believe that an approach which maintains itself at this quantitative level is the best suited for removing, as much as possible, the conceptual ambiguities that inevitably arise from such a wide and complex subject. To pursue our goal, we present the subject starting from the main ideas of physics (classical, quantum and statistical physics) relevant to the subsequent description of chemically-interesting phenomena. These ideas are analyzed systematically, trying to avoid any confusion between assumptions and logical conclusions. Our task has been to write a book which is not a mere explanation of apparently unrelated results, but an individual presentation of the essence of a connected theory, with no claim of completeness. In fact, our hope is that the book will not be used or judged for completeness, but for organization, clarity and economy.

The question arises whether the book is suited to students. A potential concern about our approach might be that it could encourage students to doubt their intuition. However, chemical-physics concepts often require 
very simple mathematics and logic, as opposed to intuition. Of course, intuition is important in the subject, but, in our opinion, it is unwise to avoid mathematics and logic, since they strengthen rather than compete with intuition. Moreover, the very essence of chemical physics is the task of eventually fitting any result or concept relying on intuition within the more satisfactory rigor of a formal conceptual structure. Therefore, the answer to the above question is that the book is indeed intended to be useful to students, not only in chemistry, but also in those fields (physics, materials science, engineering, biology) where there is a need for a knowledge of chemistry which includes, besides the presentations of the facts, their explanation in terms of general principles.

We have found some major weaknesses in the available literature on the subject. First of all, there appears to be a lack of continuity between introductory books and the sophisticated concepts needed for the more quantitative approach we have been looking for. The applied books give at most a brief discussion of background material, since their objective is not to use the applications to strenghten the reader's understanding of basic concepts, but instead to provide a detailed discussion of special tcpics. As for the most advanced books, they are often lengthy onethousand-page treatises, more suitable as informative reference books than formative tools.

The book has been written to be read in consecutive order. However, two alternatives may be followed: (i) sections 3.4-3.6 may be postponed after chapter 5, and (ii) sections 6.4-7.2.1 may be read right after chapter 3 . Consistent with the spirit of this book (which focuses on fundamentals rather than applications), we suggest that the reader, as a useful exercise, fills in the missing steps as we go from a given equation to the next one (and continues to do this for all the equations or, at least, for most of them). The notation used is very common, and should create no difficulty to the reader. For instance, vectors are written in boldface and their components are labeled by Latin letters $(j, k, \ldots=1,2,3)$.

Most of the assertions in chapters 3-7 have been proved, whereas many in chapters 1-2 have been only quoted. The reason is that all assertions of the first two chapters have been proved in our book Lecture Notes in Classical and Quantum Physics (Blackwell Scientific Publications, Oxford, 1990), to which the interested reader might refer.

One of us (FB) aknowledges Professors Arieh Ben-Naim, Augusto Rastelli and Luisa Schenetti for valuable discussions, and the Department of Chemistry at the Università di Modena, Italy, for their kind hospitality while this work was completed. 